\title{
Individual Patient Data Meta-Analysis of the Value of Microsatellite Instability As a Biomarker in Gastric Cancer
}

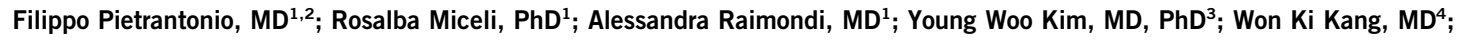 \\ Ruth E. Langley, MD, PhD ${ }^{5}$; Yoon Young Choi, MD ${ }^{6}$; Kyoung-Mee Kim, MD, PhD ${ }^{4}$; Matthew Guy Nankivell, $\mathrm{MSc}^{5}$; Federica Morano, MD; \\ Andrew Wotherspoon, $\mathrm{MBBCh}^{8}$; Nicola Valeri, MD, PhD ${ }^{8,9}$; Myeong-Cherl Kook, MD, PhD ${ }^{3}$; Ji Yeong An, MD, PhD ${ }^{4}$; \\ Heike I. Grabsch, MD, PhD, MBA ${ }^{10,11}$; Giovanni Fucà, MD ${ }^{1}$; Sung Hoon Noh, MD, PhD ${ }^{6}$; Tae Sung Sohn, PhD ; Sung Kim, MD;

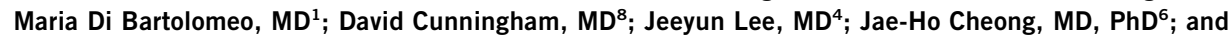 \\ Elizabeth Catherine Smyth, MD ${ }^{11}$
}

PURPOSE In the CLASSIC and MAGIC trials, microsatellite instability (MSI)-high status was a favorable prognostic and potential negative predictive factor for neoadjuvant/adjuvant chemotherapy in resectable gastric cancer (GC). Given the low prevalence of MSI-high status in GC and its association with other positive prognostic variables, large data sets are needed to draw robust evidence of its prognostic/predictive value.

PATIENTS AND METHODS We performed a multinational, individual-patient-data meta-analysis of the prognostic/ predictive role of MSI in patients with resectable GC enrolled in the MAGIC, CLASSIC, ARTIST, and ITACA-S trials. Prognostic analyses used multivariable Cox models (MVM). The predictive role of MSI was assessed both in an all-comer population and in MAGIC and CLASSIC trials by MVM testing of the interaction of treatment (chemotherapy plus surgery $v$ surgery) with MSI.

RESULTS MSI status was available for 1,556 patients: 121 (7.8\%) had MSI-high status; 576 were European, and 980 were Asian. In MSI-high versus MSI-low/microsatellite stable (MSS) comparisons, the 5-year disease-free survival (DFS) was $71.8 \%(95 \% \mathrm{Cl}, 63.8 \%$ to $80.7 \%$ ) versus $52.3 \%$ (95\% $\mathrm{Cl}, 49.7 \%$ to $55.1 \%)$; the 5 -year overall survival (OS) was $77.5 \%(95 \% \mathrm{Cl}, 70.0 \%$ to $85.8 \%$ ) versus $59.3 \%(95 \% \mathrm{Cl}, 56.6 \%$ to $62.1 \%)$. In MVM, MSI was associated with longer DFS (hazard ratio [HR], 1.88; $95 \% \mathrm{Cl}, 1.28$ to $2.76 ; P<.001$ ) and OS (HR, 1.78; $95 \% \mathrm{Cl}, 1.17$ to $2.73 ; P=.008$ ), as were $\mathrm{pT}, \mathrm{pN}$, ethnicity, and treatment. Patients with MSI-low/MSS GC benefitted from chemotherapy plus surgery: the 5 -year DFS compared with surgery only was $57 \%$ versus $41 \%$ (HR, $0.65 ; 95 \% \mathrm{Cl}, 0.53$ to 0.79 ), and the 5 -year OS was $62 \%$ versus $53 \%$ ( $\mathrm{HR}, 0.75 ; 95 \% \mathrm{Cl}, 0.60$ to 0.94 ). Conversely, those with MSI-high GC did not: the 5 -year DFS was $70 \%$ versus $77 \%(\mathrm{HR}, 1.27 ; 95 \% \mathrm{Cl}, 0.53$ to 3.04), and the 5-year OS was 75\% versus 83\% (HR, 1.50; 95\% Cl, 0.55 to 4.12).

CONCLUSION In patients with resectable primary GC, MSI is a robust prognostic marker that should be adopted as a stratification factor by clinical trials. Chemotherapy omission and/or immune checkpoint blockade should

ASSOCIATED CONTENT

Listen to the podcast by Dr Rahma at http://ascopubs.org/ jco/podcasts

Data Supplement Author affiliations and support information (if applicable) appear at the end of this article.

Accepted on August 6, 2019 and published at jco.org on

September 12, 2019: DOI https://doi.org/10. 1200/JC0.19.01124 be investigated prospectively in MSI-high GCs according to clinically and pathologically defined risk of relapse.

$\mathrm{J}$ Clin Oncol 37:3392-3400. $\odot 2019$ by American Society of Clinical Oncology

\section{INTRODUCTION}

Gastric cancer (GC) is the third most common cause of cancer-related deaths globally; almost 1 million cases are diagnosed worldwide annually. ${ }^{1}$ Treatment of patients with locally advanced resectable GC displays significant geographic variation; in Asia, surgery plus adjuvant chemotherapy is preferred, whereas, outside of Asia, perioperative chemo(radio)therapy is a more frequent choice. Adjuvant and perioperative chemotherapy are both evidence-based, guideline-endorsed treatments for GC. However, more than half of patients still experience relapse and die as a result of their disease. ${ }^{2-7}$ Conversely, some patients are cured by surgery alone and do not benefit from potentially toxic chemotherapy treatment. Current chemotherapy approaches are based on patient-related factors and clinicopathologic staging, and there is only one recently validated biomarker to select patients with GC for chemotherapy in the adjuvant setting. This prognostic and predictive tool, which is based on the expression levels of four candidate classifier genes (GZMB, WARS, SFRP4, and CDX1), was developed in Asian patients with GC who were treated in routine practice with surgery alone or surgery followed by adjuvant fluorouracil-based chemotherapy; it was validated on a cohort of patients enrolled in the CLASSIC trial and showed promising results. ${ }^{8}$

\section{ASCO}


Mismatch repair deficiency and microsatellite instability (MSI) has been associated with improved survival in colon cancer and may be predictive of lack of benefit from adjuvant chemotherapy in stage $\|$ disease. ${ }^{9,10}$ As a result, assessment of mismatch repair deficiency by immunohistochemistry or MSI is recommended for patients with resected colorectal cancer to guide treatment decisions and screen for Lynch syndrome. ${ }^{11,12}$ Results from two pivotal clinical trials have suggested a similar relationship between $\mathrm{MSI}$ and outcomes in patients with curatively resected GC. In the MAGIC trial, which established perioperative chemotherapy as a standard of care for nonAsian patients with $\mathrm{GC},{ }^{3} \mathrm{MSI}$-high status, compared with a microsatellite stable (MSS)/MSI-low subgroup, had an improved prognosis in the surgery-alone treatment arm (hazard ratio [HR], $0.35 ; 95 \% \mathrm{Cl}, 0.11$ to $1.11 ; P=.08$ ) and a worse survival outcome in the chemotherapy-plus-surgery arm (HR, 2.22; 95\% Cl, 1.02 to $4.85 ; P=.04) .{ }^{13}$ In the CLASSIC trial, which established adjuvant capecitabine and oxaliplatin as a standard for Asian patients with resectable GC disease, ${ }^{2}$ patients with MSI-high GC did not derive any survival benefit from adjuvant chemotherapy (5-year disease-free survival [DFS] for chemotherapy $v$ surgery only groups: $83.9 \% \vee 85.7 \% ; P=.93) .{ }^{14}$

Despite these results from historically important randomized clinical trials (RCTs), a significant challenge for the adoption of $\mathrm{MSI} /$ mismatch repair deficiency testing as a routine biomarker in patients with operable GC is the low MSI prevalence in GC ( $8 \%$ to $10 \%)$, which led to limited statistical power of the observations in individual trial data sets. ${ }^{13-15}$ With this in mind, we pooled individual patient data from four large, multinational RCTs performed in patients with resectable GC (MAGIC, ${ }^{3} \mathrm{CLASSIC},{ }^{2}$ ARTIST, ${ }^{16}$ and ITACA-S ${ }^{17}$ ), and evaluated the relationship between MSI status, overall survival (OS), and DFS and biomarker interaction with chemo(radio)therapy treatment.

\section{PATIENTS AND METHODS}

\section{Study Design and Trial Populations}

We performed an individual patient data (IPD) metaanalysis from four multicenter RCTs in patients with resectable GC: the MAGIC, CLASSIC, ITACA-S, and ARTIST trials. Two trials investigated whether the addition of perioperative or adjuvant chemotherapy to radical surgery could improve the survival in this patient population. In the MAGIC ${ }^{3}$ trial, patients were randomly allocated to perioperative chemotherapy (epirubicin, cisplatin, and fluorouracil) plus surgery or to surgery alone, whereas, in the CLASSIC $^{2}$ trial, patients who had received radical surgery were randomly assigned to follow-up or adjuvant chemotherapy with capecitabine plus oxaliplatin. In the ITACA-S ${ }^{17}$ trial, patients were randomly assigned to receive two different schedules of adjuvant chemotherapy: an intensified combination chemotherapy schedule (fluorouracil plus leucovorin plus irinotecan followed by cisplatin plus docetaxel) or single-agent chemotherapy (fluorouracil plus leucovorin). In the ARTIST ${ }^{16}$ trial, patients were randomly assigned to adjuvant chemotherapy (capecitabine plus cisplatin) versus chemotherapy (capecitabine plus cisplatin) plus concurrent irradiation.

Comprehensive eligibility criteria and complete results of each trial have been previously published. ${ }^{2,3,16,17}$ In addition, post hoc analyses of all trials aimed at investigation of the interaction between MSI status and patient outcome have already been presented. ${ }^{13-15,18}$ The purpose of this study was to assess the potential prognostic role of MSI status in patients with radically resected GC and its potential value to predict the outcome to systemic treatment in this patient population.

\section{Data Gathering}

The analysis was designed in 2018. The members of all trial management committees gave their approval according to a formal protocol.

The requested data consisted of patient characteristics (including age, sex, ethnicity, Eastern Cooperative Oncology Group performance status, tumor localization, T and N stage reclassified according to the American Joint Committee on Cancer seventh edition TNM staging manual, ${ }^{19}$ histology type according to Lauren classification, and treatment arm classified as multimodal treatment $v$ surgery only), MSI status (MSI-low/MSS versus MSI-high) and outcome data (disease relapse and survival). Patients with unknown/not assessed MSI status were excluded. For detailed methodology on MSI assessment in each trial, see the Data Supplement.

A trial database was set up to include the information extrapolated by the four studies datasets. This ensured the collection of appropriately comparable data, which thus allowed the planned IPD pooled analysis. All patients had given informed consent for trial participation, and this study was approved by the ethical committee of Fondazione IRCCS Istituto Nazionale dei Tumori.

\section{Statistical Analysis}

Trial heterogeneity according to patient and disease characteristics was tested by using the Kruskal-Wallis test for numeric variables or the Fisher-Freeman-Halton Fisher test for categoric variables. ${ }^{20}$ To investigate dishomogeneity between MSI status categories, we used the Mann-WhitneyWilcoxon test for numeric variables or the Fisher-FreemanHalton Fisher test for categoric variables.

DFS and OS curves were estimated with the Kaplan-Meier method. Between-group differences were tested with the log-rank test.

The prognostic role of MSI was assessed in the whole population. IPD multivariable analyses were performed using Cox models that included the trial as a random variable. The multivariable models, together with MSI, included the following variables (covariables): patient characteristics (age, sex, ethnicity), Eastern Cooperative Oncology Group 
performance status, tumor localization, $\mathrm{T}$ and $\mathrm{N}$ stage, histology, and treatment arm. We also fitted random effect models with inverse variance weighting to pool the MSI HRs estimated from trial-level univariable Cox models.

The predictive role of MSI was assessed in multivariable Cox models with trial as a random variable, using IPD both from the four trials and from the two trials with a surgery-alone arm (MAGIC + CLASSIC). The models included all the above covariables and the interaction of treatment arm by MSI.

Age was modeled as a continuous variable using three-knot restricted cubic spline,$^{21}$ and the other covariates as categorical using dummy variables. In the multivariable analyses, patients with missing covariable data were excluded. The analyses were performed using SAS version 9.2 (SAS Institute, Cary, NC) and R software.

\section{RESULTS}

Overall, 2,648 patients were included in the initial trial database; 1,092 patients were excluded from the study as per prespecified criteria (did not have radical surgery for the primary tumor and lymph nodes or MSI status unknown or not assessed). Therefore, a total of 1,556 patients were included in the final data set of the pooled analysis (317 from MAGIC, 592 from CLASSIC, 259 from ITACA-S, and 388 from ARTIST trial; Fig 1). The selected population from each trial was not notably different from the corresponding whole-trial population, as reported previously. ${ }^{13-15,22}$

Baseline patients and disease characteristics are listed in the Data Supplement. Briefly, two trials (CLASSIC and ARTIST) were conducted in Asian countries, and two others (MAGIC and ITACA-S) were performed on a European population. In the overall study population, the median age was 59 years (range, 20 to 85 years), and Asian and European ethnicities accounted for $63 \%$ and $37 \%$, respectively, of the population. Most patients were men $(70.1 \%)$, and most tumors were located in the stomach versus distal esophageal/gastroesophageal junction (91.7\% v 8.3\%). Intestinal histology represented $45.1 \%$, and other histotypes were $54.9 \%$ of the whole-trial population. Overall, 1,101 patients $(70.8 \%)$ received multimodal treatment, whereas 455 (29.2\%) were treated with surgery only. The Data Supplement shows the KaplanMeier curves of DFS and OS in the four trial populations, and it shows the corresponding 5-year estimates.

The frequency of MSI-high GCs was well balanced between the four trials and in the two treatment arms (multimodal treatment $v$ surgery only). In the MSI-high subgroup versus the MSS/MSI-low subgroup, the median age was 66 versus 58 years, and the prevalence of intestinal type histology was $67.5 \%$ versus $43.2 \%$ (both $P<.001$ ), whereas gastric tumor localization was $96.7 \%$ versus $91.3 \%(P=.056$; Table 1). When patients with MSI-high versus MSS/MSI-low GC were compared, the 5-year DFS was $71.8 \%(95 \% \mathrm{Cl}$, $63.8 \%$ to $80.7 \%$ ) versus $52.3 \%$ (95\% $\mathrm{Cl}, 49.7 \%$ to $55.1 \%$ ), which included 32 versus 678 events $(P<.001$; Fig 2A), and the 5 -year OS was $77.5 \%(95 \% \mathrm{Cl}, 70.0 \%$ to $85.8 \%)$ versus $59.3 \%$ ( $95 \% \mathrm{Cl}, 56.6 \%$ to $62.1 \%)$, which included 26 versus 566 events $(P<.001$; Fig 2B).

In the multivariable prognostic IPD analyses (Data Supplement), MSI was independently associated with DFS (HR for MSS/MSI-low $v$ MSI-high status, 1.88; $95 \% \mathrm{Cl}$, 1.28 to $2.76 ; P=.001$ ) and $\mathrm{OS}(\mathrm{HR}, 1.78 ; 95 \% \mathrm{Cl}, 1.17$ to 2.73; $P=.008)$. Other significant factors for survival were ethnicity (worse prognosis associated with European), $\mathrm{T}$ and $\mathrm{N}$ stage (by AJCC, seventh edition ${ }^{19}$ ), and treatment arm (worse prognosis associated with surgery only), whereas sex was significantly associated with OS only (worse prognosis associated with men). To compare these MSI results with those from the classical meta-analytic approach on the basis of aggregate data from each trial, we estimated the pooled HR from trial-level univariable Cox models ( $P=.010$ for both DFS and OS; Data Supplement). Because of the heterogeneity of MSI effect between the trials and the dishomogeneity of patient characteristics, we obtained more imprecise HR estimates (larger Cls) compared with the IPD result. In addition, although the DFS HR was slightly lower in the aggregate data analysis than in the IPD analysis ( $1.80 v 1.88)$, the OS HR was slightly higher ( 1.88 v 1.78).

We also investigated whether the MSI prognostic effect could vary according to ethnicity by fitting multivariable Cox models to include the interaction of MSI by ethnicity. This interaction was statistically significant for both DFS $(P=.041)$ and OS $(P=.035)$, which translates in a greater MSI prognostic effect in Asian patients (HR for MSS/MSIlow $v$ MSI-high status, 2.83; $95 \% \mathrm{Cl}, 1.55$ to 5.15 for DFS and $\mathrm{HR}, 2.97 ; 95 \% \mathrm{Cl}, 1.46$ to 6.04 for OS) than in European patients (HR, 1.26; $95 \% \mathrm{Cl}, 0.76$ - to 2.07 for DFS and $\mathrm{HR}, 1.15 ; 95 \% \mathrm{Cl}, 0.67$ to 1.96 for OS; other data not shown). The Data Supplement shows the Kaplan-Meier DFS and OS curves according to $\mathrm{N}$ and T stage.

In the whole-population analyses of the predictive role of MSI (Table 2; Figs 3A and 4A), the interaction of treatment by MSI was not statistically significant for either DFS and OS ( $P=.133$ and .180 , respectively). However, only patients with MSS/MSI-low GC had a significant benefit for chemotherapy versus surgery only: the 5-year DFS was 57\% versus $41 \%(\mathrm{HR}, 0.65 ; 95 \% \mathrm{Cl}, 0.53$ to 0.79$)$, and the 5 year OS was $62 \%$ versus $53 \%$ (HR, $0.75 ; 95 \% \mathrm{Cl}, 0.60$ to 0.94). Patients with MSI-high tumors had a 5-year DFS of $70 \%$ versus $77 \%$ in $\mathrm{MSS} / \mathrm{MSI}-$ low subgroup (HR, 1.27; $95 \% \mathrm{Cl}, 0.53$ to 3.04 ) and a 5 -year OS of $75 \%$ versus $83 \%$, respectively (HR, 1.50; $95 \% \mathrm{Cl}, 0.55$ to 4.12$)$. The corresponding results in the MAGIC and CLASSIC trials are listed in Table 2 and Figures 3B and 4B.

Finally, we performed a survival after recurrence (SAR) analysis in the whole-trial population stratified according to MSI status. We did not find a significant difference in terms 


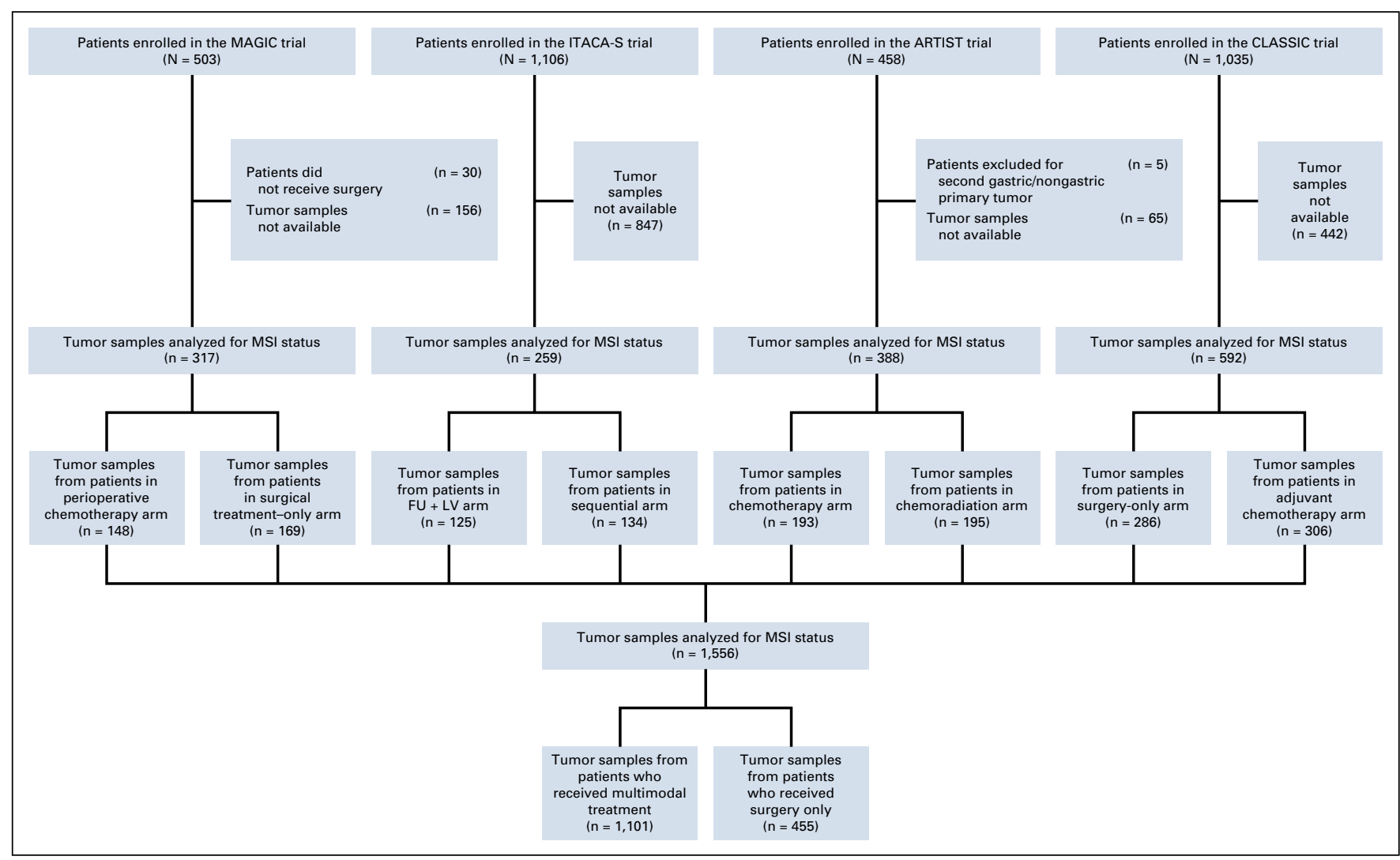

FIG 1. Flow diagram of patient selection for the meta-analysis. FU, fluorouracil; LV, leucovorin; MSI, microsatellite instability.

of SAR in MSI-high versus MSS/MSI-low subgroups: the 12-month SAR was $33.0 \%$ and $35.9 \%$, respectively (HR, $1.01 ; 95 \% \mathrm{Cl}, 0.68$ to $1.50 ; P=.947$; data not shown).

\section{DISCUSSION}

The results of this meta-analysis confirm the positive prognostic role of MSI in surgically resected GC and suggest a potential lack of benefit of perioperative or adjuvant chemotherapy for patients with MSI-high GC who undergo surgery. These findings are notable, because they are consistent with the results of individual clinical trials that alone were underpowered. ${ }^{13,14}$ Pooling of the individual patient data from the four RCTs has provided clinically robust and more generalizable results about the prognostic and predictive value of MSI. Moreover, we show the limitations of meta-analyses that are based on summary data extracted from trial reports (Data Supplement) and show that more precise estimates of MSI HRs can be obtained by multivariable modeling of IPD data that considers patients and trial heterogeneity. Finally, we also were able to perform additional subgroup analyses to provide more insights from IPD data. On the basis of these results, we suggest that, in patients with MSI-high GC that is resectable, careful multidisciplinary discussion should be adopted in light of the overall prognostic assessment and potential harm from systemic chemotherapy. Patients with MSI-high GC represent up to $10 \%$ of operable GC occurrences, so this has implications for many thousands of patients annually globally. ${ }^{1}$

In our prognostic analyses, we evaluated the relationship between MSI status and outcome in patients with treated $\mathrm{GC}$ in all four trials. Our meta-analysis demonstrated a large benefit from chemotherapy in patients with MSS/MSI-low status with respect to DFS and OS and suggests a lack of benefit from chemotherapy in patients with MSI-high status (HR estimates are listed in Table 2). The interaction test for differential outcome according to biomarker status in patients with chemotherapy- and non-chemotherapy-treated disease was not significant in the entire cohort; however, given the small size of the MSI-high subgroup and the consequent low number of events, the interaction test could be quite underpowered. We also investigated the predictive effect of MSI when limited to the MAGIC and CLASSIC trials. These were the only trials that contributed surgery-only arms; in this group of patients, the $P$ values for the treatmentby-MSI interaction (.147 for DFS and .070 for OS) became closer to the conventional 5\% significance level. However, the caveats of small subgroup analyses also still apply.

Consistent with previous findings, elderly patients were diagnosed more frequently with MSI-high GC. ${ }^{23}$ There was no female patient preponderance. Our results also confirm that MSI-high tumors are more often found in the stomach rather than the gastroesophageal junction or lower 
TABLE 1. Patient and Disease Characteristics According to MSI Status

\section{No. (\%) of Patients}

\begin{tabular}{|c|c|c|c|c|}
\hline & & & & \\
\hline Characteristic & $\begin{array}{l}\text { Whole Series } \\
(\mathrm{N}=1,556)\end{array}$ & $\begin{array}{l}\text { MSI High } \\
(n=121)\end{array}$ & $\begin{array}{c}\text { MSI Low/MSS } \\
(n=1,435)\end{array}$ & $P$ \\
\hline Trial & & & & .313 \\
\hline MAGIC & $317(20.4)$ & $21(17.4)$ & $296(20.6)$ & \\
\hline CLASSIC & $592(38.0)$ & $40(33.0)$ & $552(38.5)$ & \\
\hline ITACA-S & $259(16.7)$ & $25(20.7)$ & $234(16.3)$ & \\
\hline ARTIST & $388(24.9)$ & 35 (28.9) & $353(24.6)$ & \\
\hline Median (range) age, years & $59(20-85)$ & $66(32-77)$ & $58(20-85)$ & $<.001$ \\
\hline Sex & & & & .835 \\
\hline Male & $1,091(70.1)$ & $86(71.1)$ & $1,005(70.0)$ & \\
\hline Female & 465 (29.9) & 35 (28.9) & $430(30.0)$ & \\
\hline Ethnicity & & & & .846 \\
\hline White & $576(37.0)$ & $46(38.0)$ & 530 (36.9) & \\
\hline Asian & $980(63.0)$ & $75(62.0)$ & $905(63.1)$ & \\
\hline ECOG PS & & & & .495 \\
\hline 0 & $1,205(77.7)$ & $91(75.2)$ & $1,114(77.9)$ & \\
\hline 1 & $346(22.3)$ & $30(24.8)$ & $316(22.1)$ & \\
\hline Not available & 5 & 0 & 5 & \\
\hline Tumor localization & & & & .056 \\
\hline Esophageal, distal/GEJ & $128(8.3)$ & $4(3.3)$ & $124(8.7)$ & \\
\hline Gastric & $1,422(91.7)$ & $116(96.7)$ & $1,306(91.3)$ & \\
\hline Not available & 6 & 1 & 5 & \\
\hline Histology & & & & $<.001$ \\
\hline Intestinal & $678(45.1)$ & 79 (67.5) & 599 (43.2) & \\
\hline Other & 825 (54.9) & $38(32.5)$ & 787 (56.8) & \\
\hline Not available & 53 & 4 & 49 & \\
\hline T stage ${ }^{*}$ & & & & .192 \\
\hline 1 & $96(6.5)$ & $7(6.0)$ & 89 (6.6) & \\
\hline 2 & 285 (19.3) & $17(14.7)$ & 268 (19.7) & \\
\hline 3 & 577 (39.1) & $56(48.3)$ & 521 (38.3) & \\
\hline 4 & $517(35.1)$ & $36(31.0)$ & $481(35.4)$ & \\
\hline Not available & 81 & 5 & 76 & \\
\hline N stage* & & & & .225 \\
\hline 0 & 185 (11.9) & 14 (11.6) & 171 (11.9) & \\
\hline 1 & 441 (28.3) & $43(35.5)$ & $398(27.7)$ & \\
\hline 2 & $473(30.4)$ & $34(28.1)$ & 439 (30.6) & \\
\hline $3 a$ & 379 (24.4) & $28(23.1)$ & 351 (24.5) & \\
\hline $3 b$ & $78(5.0)$ & $2(1.7)$ & $76(5.3)$ & \\
\hline Treatment arm & & & & .678 \\
\hline Chemotherapy & $1,101(70.8)$ & $88(72.7)$ & 1,013 (70.6) & \\
\hline Surgery & 455 (29.2) & $33(27.3)$ & $422(29.4)$ & \\
\hline
\end{tabular}

NOTE. $P$ values are from Mann-Whitney-Wilcoxon test for numeric variables or Fisher-Freeman-Halton Fisher test for categoric variables.

Abbreviations: ECOG, Eastern Cooperative Oncology Group; GEJ, gastroesophageal junction; MSI, microsatellite instability; MSS, microsatellite stable; PS, performance status.

${ }^{*} \mathrm{~T}$ and $\mathrm{N}$ stages are according to American Joint Committee on Cancer 7th edition TNM staging system. ${ }^{19}$ 


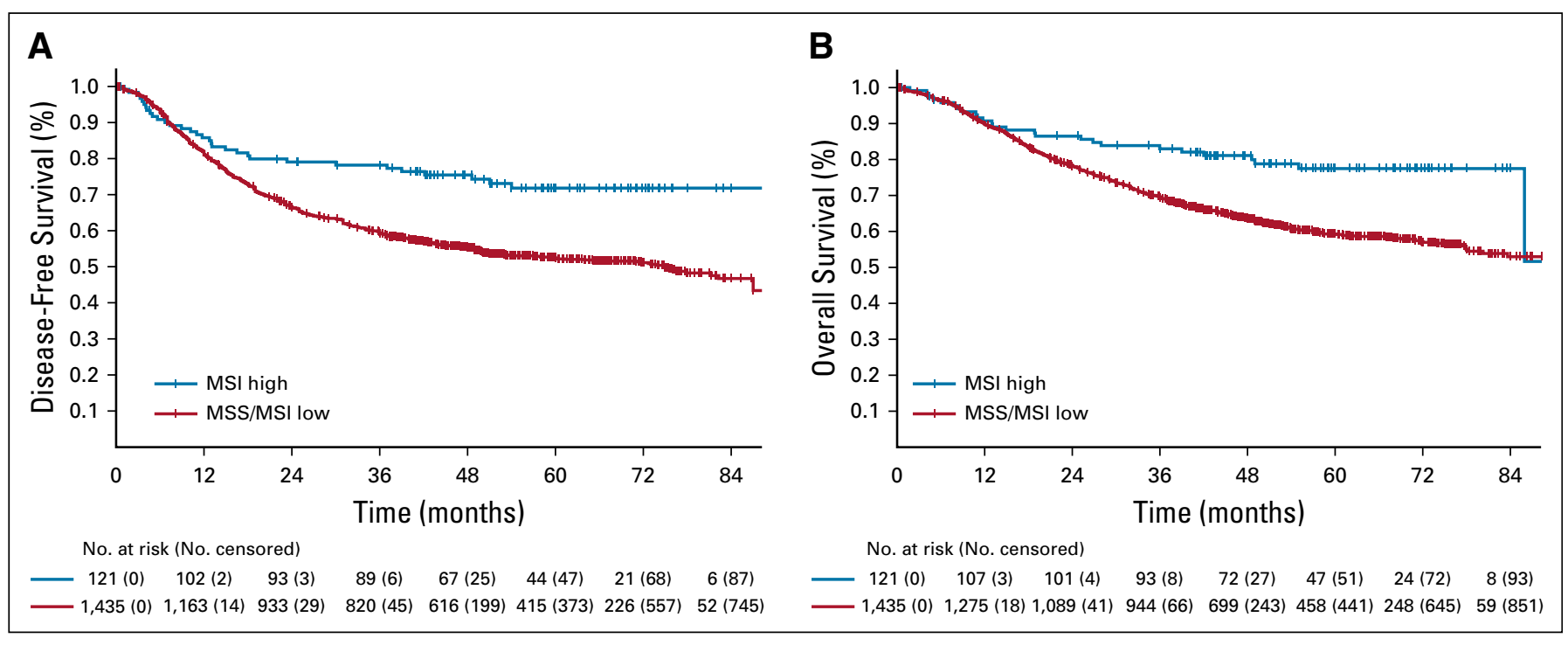

FIG 2. Kaplan-Meier curves of (A) disease-free survival and (B) overall survival according to microsatellite-instability (MSI) status (microsatellite stable [MSS]/ MSI-low $v$ MSI-high).

esophagus ( $9 \%$ and $3 \%$ in MSS/MSI-low and MSI-high groups, respectively) and are more often the Lauren classification's intestinal subtype. ${ }^{23}$ However, we did not find that MSI was enriched in early-stage cancers, as has been suggested. ${ }^{24}$ This could be accounted for by stringent trial eligibility criteria that required enrollment of predominantly more locally advanced cancers. However, the results of this multivariable analysis demonstrate that MSI is prognostic independent of $\mathrm{T}$ and $\mathrm{N}$ stage. This may imply that, even in GCs with more advanced disease stage, it might be possible to forego chemotherapy for patients who have operable MSI tumors. Moreover, given the evidence to support a poor prognostic effect of MSI-high status in patients with advanced/metastatic colorectal cancer, we investigated whether, in our pooled analysis in the GC setting, a similar result could be observed. ${ }^{25,26}$ No significant differences were reported in terms of survival after recurrence in the MSI-high subgroup compared with MSS/ MSI-low subgroup, although the reliability of these data are limited by the small number of patients who experienced disease progression in the MSI-high subgroup. In addition, because all patients who have metastatic GC have limited survival compared with patients who have metastatic colorectal cancer, differences in the outcome may be more difficult to measure. Moving forward, in the era of immunotherapy, it will be challenging to perform additional analyses on the topic of

TABLE 2. Analyses of MSI Predictive Role: Impact of Chemotherapy in MSI-High and MSS/MSI-Low Subgroups

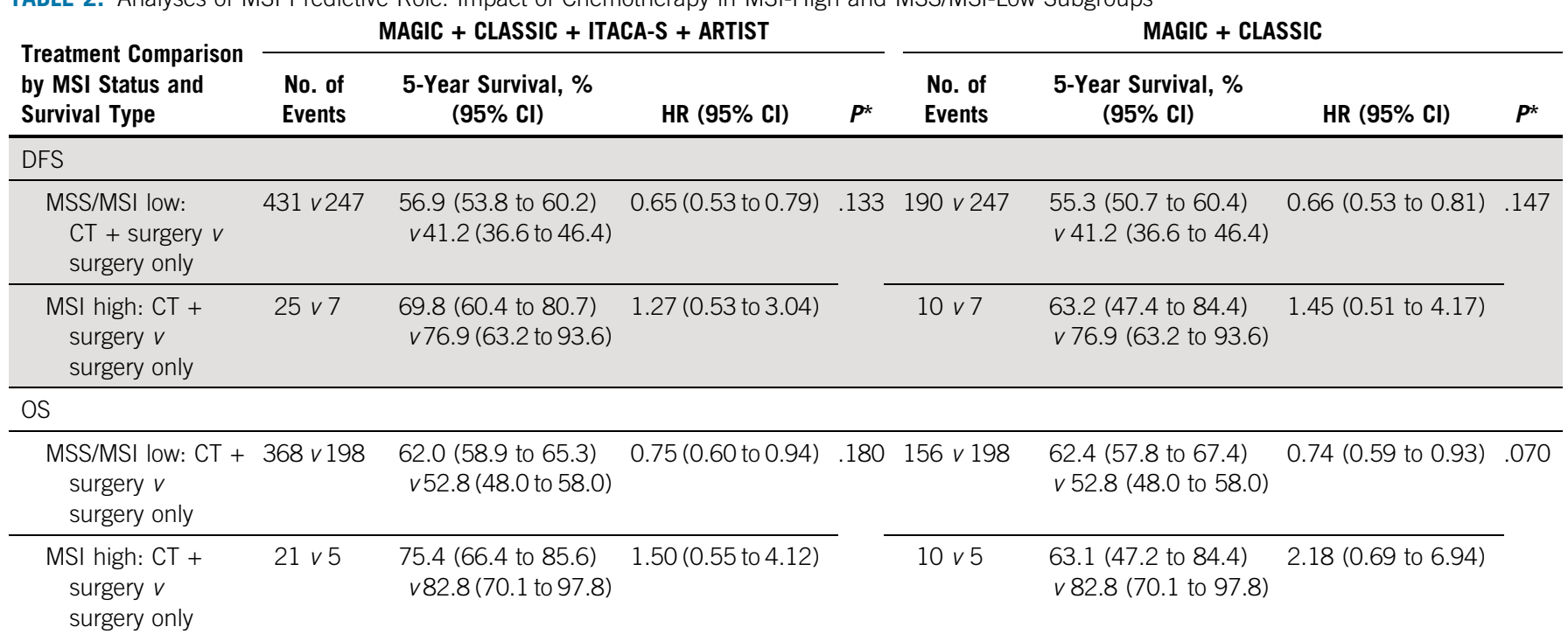

NOTE. Wald test $P$ value was used to test interaction treatment by MSI.

Abbreviations: CT, chemotherapy; DFS, disease-free survival; HR, hazard ratio; MSI, microsatellite instability; MSS, microsatellite stable; OS, overall survival. 


\section{A}

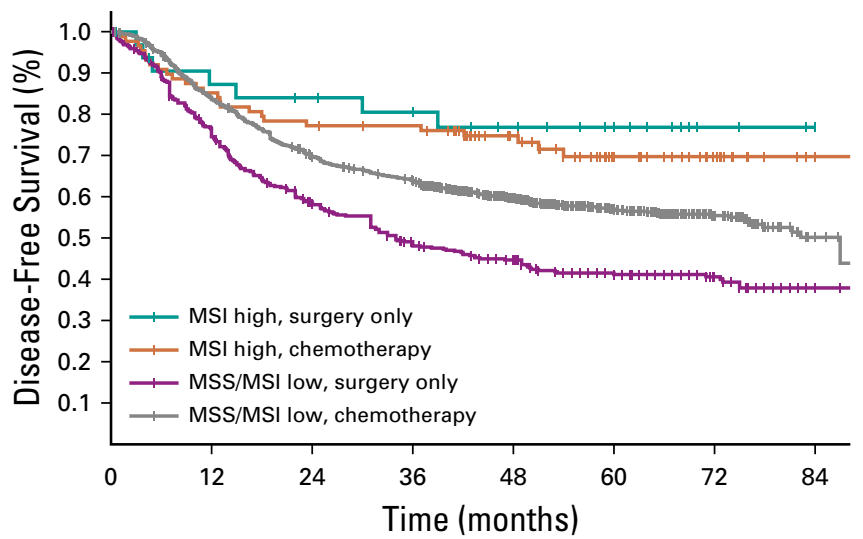

No. at risk (No. censored)

$\begin{array}{cccccccc}33(0) & 27(2) & 25(3) & 23(4) & 19(7) & 15(12) & 4(22) & 1(26) \\ -88(0) & 75(0) & 68(0) & 66(2) & 48(18) & 29(35) & 17(46) & 5(61) \\ -422(0) & 318(6) & 238(13) & 192(20) & 163(34) & 115(72) & 68(119) & 16(172) \\ -1,013(0) & 845(8) & 695(16) & 628(25) & 453(165) & 300(301) & 158(438) & 36(573)\end{array}$
B

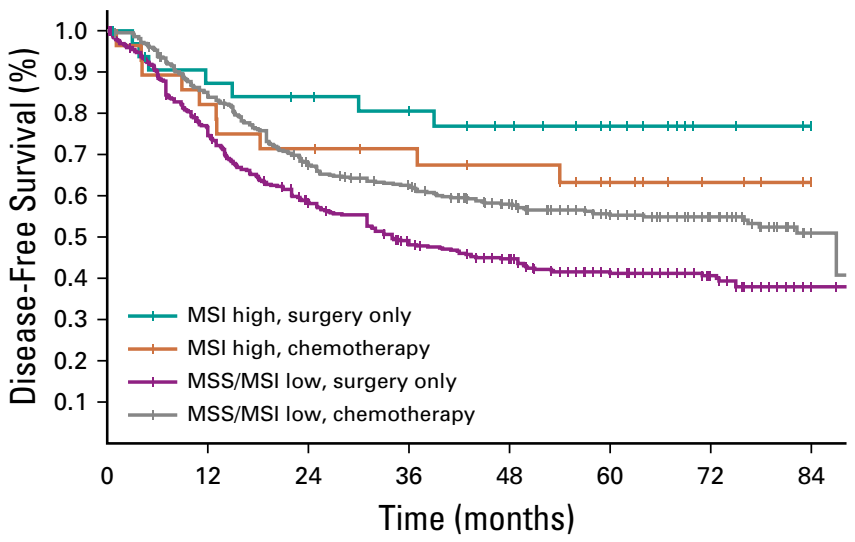

No. at risk (No. censored)

$\begin{array}{cccccccc}33(0) & 27(2) & 25(3) & 23(4) & 19(7) & 15(12) & 4(22) & 1(26) \\ 28(0) & 23(0) & 20(0) & 18(2) & 16(3) & 12(7) & 7(11) & 3(18) \\ -422(0) & 318(6) & 238(13) & 192(20) & 163(34) & 115(72) & 68(119) & 16(172) \\ -426(0) & 358(5) & 281(10) & 252(17) & 211(43) & 164(83) & 105(142) & 31(232)\end{array}$

FIG 3. Kaplan-Meier curves of disease-free survival according to treatment (surgery plus chemotherapy $v$ surgery only) and microsatellite-instability (MSI) status (MSI-high $v$ microsatellite stable [MSS]/MSI-low) in (A) whole trial population and (B) MAGIC and CLASSIC trials only.

SAR, because most patients with MSI-high metastatic GC would receive an immune checkpoint inhibitor, which potentially changes the natural history of this disease subgroup. ${ }^{27}$

The results of the multivariable analyses also highlight the superior survival of Asian compared with European patients (HR, 4.38 for European $v$ Asian ethnicity; $P=.002$ ). This is well recognized and has potentially confounded the results of international GC trials. ${ }^{28,29}$ Unexpectedly, the positive prognostic effect of MSI status was more pronounced in
Asian than European patients (HR for DFS and OS, 2.83 and $2.97 v 1.26$ and 1.15 , respectively). This could be explained by the negative results in chemotherapy-treated patients in the MAGIC trial; however, similar findings are noted in ITACA$S$ and ARTIST, neither of which have nonchemotherapy control groups. It is possible that either the driver state (tumor mutation burden) or the response to hypermutation is different between the two patient groups. It has been noted previously that the immune microenvironment in

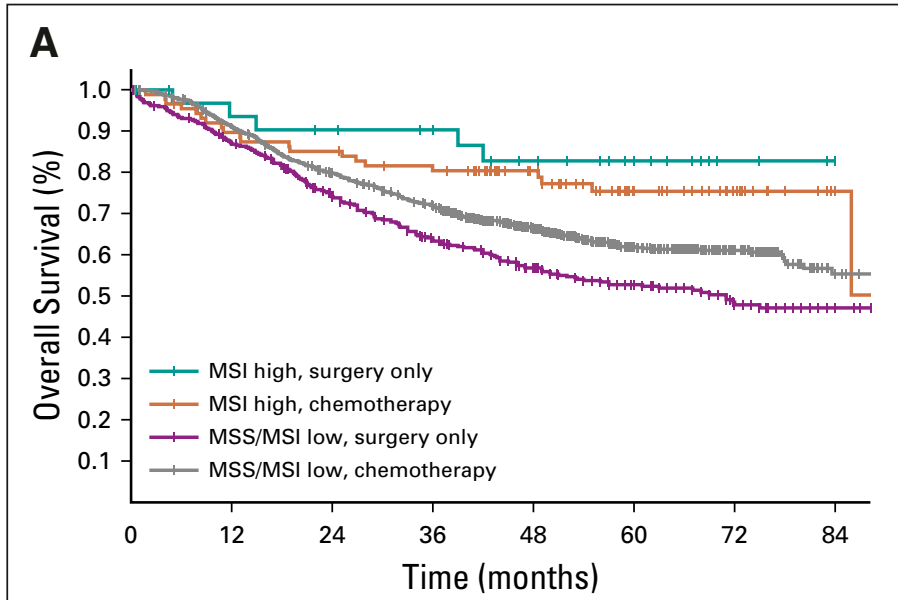

No. at risk (No. censored)

$\begin{array}{llllll}-33(0) \quad 29(2) & 27(3) \quad 25(5) \quad 20(8) & 15(14) \quad 4(24) & 1(28)\end{array}$

$\begin{array}{rlllll}-88(0) & 78(1) \quad 74(1) \quad 68(3) \quad 52(19) \quad 32(37) \quad 20(48) \quad 7(65)\end{array}$

$\begin{array}{llllllll}422(0) & 361(7) & 299(20) & 245(31) & 197(56) & 138(101) & 77(157) & 18(219)\end{array}$

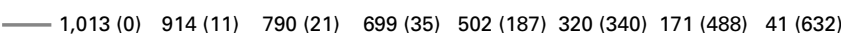

\section{B}

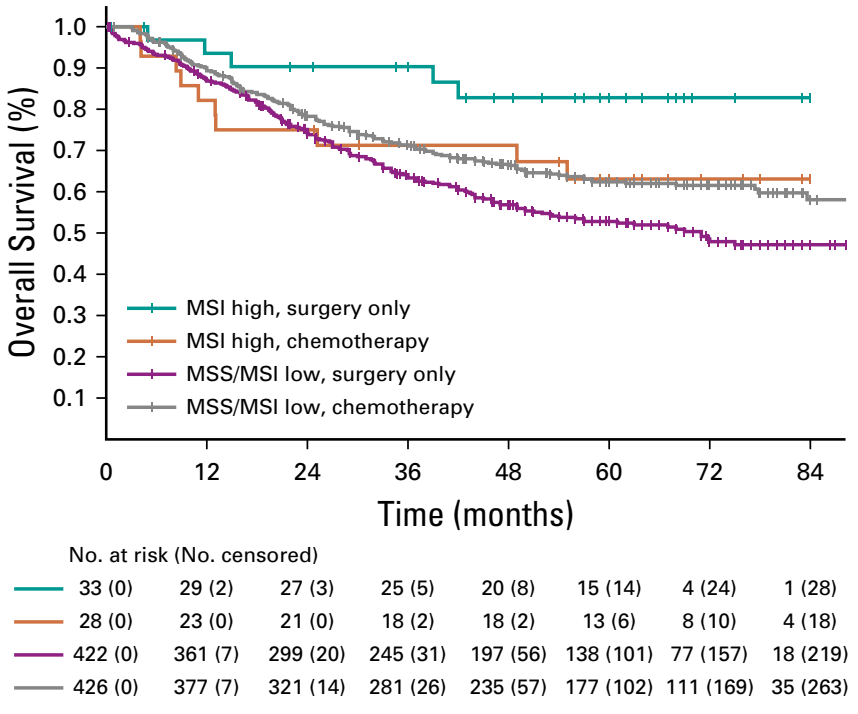

FIG 4. Kaplan-Meier curves of overall survival according to treatment (surgery plus chemotherapy $v$ surgery only) and microsatellite-instability (MSI) status (MSI-high $v$ microsatellite stable [MSS]/MSI-low) in (A) whole trial population and (B) MAGIC and CLASSIC trials only. 
Asian GC differs from non-Asian cancers. ${ }^{30}$ Additional research is required to clarify whether this is responsible for the difference in magnitude of effect of MSI in Asian patients with GC.

The major challenge to the generalizability of our results is the heterogeneity of patients included in the meta-analysis who were of European and Asian origin and who had different surgical approaches (D1 $v$ D2 resection) and treatments (neoadjuvant $v$ adjuvant chemotherapy). These differences did indeed lead to different prognoses in each cohort. However, in each individual study (and in CLASSIC and MAGIC, which had control groups), MSI had an almost identical interaction with chemotherapy. What we aimed to show was that the effect of MSI is universal, although it may be different in magnitude in selected groups because of the aforementioned reasons. Furthermore, MSI status was assessed using a widely available, standard, validated assay, which implies that integration of MSI status into standard preoperative evaluation on biopsy material may be feasible. ${ }^{31}$ Another potential criticism is that we did not assess MSI in pretreatment biopsies or by using immunohistochemistry, although we are reassured that concordance between MSI and MMRD in gastric cancer was greater than $95 \%$ in previous data sets. ${ }^{13}$ Because MSI is a fundamental characteristic of a tumor, there are few concerns that this would change in a post-treatment specimen, as was used in this study. Family history status was not available to evaluate any differential outcomes

\footnotetext{
AFFILIATIONS

${ }^{1}$ Fondazione IRCCS Istituto Nazionale dei Tumori, Milan, Italy

${ }^{2}$ University of Milan, Milan, Italy

${ }^{3}$ National Cancer Center, Goyang, Korea

${ }^{4}$ Samsung Medical Center Sungkyunkwan University School of Medicine, Seoul, Korea

${ }^{5}$ The Medical Research Council Clinical Trials Unit at University College London, London, United Kingdom

${ }^{6}$ Yonsei University College of Medicine, Seoul, Korea

${ }^{7}$ Royal Marsden Hospital, London and Sutton, United Kingdom

${ }^{8}$ The Institute of Cancer Research, London, United Kingdom

${ }^{9}$ GROW School for Oncology and Developmental Biology, Maastricht

University Medical Center+, Maastricht, The Netherlands

${ }^{10}$ Leeds Institute for Medical Research at St James's, University of Leeds, Leeds, United Kingdom

${ }^{11}$ Cambridge University Hospitals NHS Foundation Trust, Cambridge, United Kingdom
}

\section{CORRESPONDING AUTHOR}

Elizabeth C. Smyth, MD, Cambridge University Hospitals NHS Foundation Trust, Cambridge, United Kingdom; e-mail: elizabeth.smyth2@nhs.net.

\section{EQUAL CONTRIBUTION}

F.P. and R.M. contributed equally to this work.

\section{PRIOR PRESENTATION}

Presented as a poster at the ASCO Gastrointestinal Cancers Symposium, San Francisco, CA, January 23-25, 2019. in Lynch versus sporadic MSI cancers; however, in previous studies, more than $80 \%$ of MSI gastric cancers are MLH1 methylated and sporadic. ${ }^{32}$ Finally, although this data set was large ( $n=1,556$ patients), the absolute number of MSI tumors was relatively low $(n=121)$, which might be considered few when compared with comparable studies in colorectal cancer. ${ }^{33}$ However, this represented the totality of the trials in this setting that had tissue available.

In conclusion, we present for the first time, to our knowledge, the results of an IPD meta-analysis of the effect of MSI status on long-term oncologic outcome for patients with resectable GC treated in clinical trials. On the basis of our findings, we believe that patients with MSIhigh GC who are treated with surgery alone could perform well even without adjunctive chemotherapy. In contrast, patients with MSI-high GC who are treated with chemotherapy (perioperative or adjuvant) might not benefit from this treatment. We suggest that patients who have operable GC that is MSI and resectable should undergo critical multidisciplinary discussion if neoadjuvant or adjuvant chemotherapy is to be considered. Because anti-programmed death 1 antibodies are associated with response rates of greater than $50 \%$ in MSI-high advanced $\mathrm{GC},{ }^{27,34}$ the next rational step is to design a clinical trial in which patients with operable MSI-high GC are treated with neoadjuvant or adjuvant immune checkpoint blockade to improve survival even more for this biomarker-selected group of patients.

\section{SUPPORT}

There was no specific funding source for this work. ECS thanks the Cambridge Biomedical Research Center for their support. RMH authors acknowledge the support of the NIHR RM/ICR BRC. Translational work on the MAGIC trial was supported by Cancer Research UK Grant C20023/ A7217. Work on ITACA-S was supported by Associazione Italiana per la Ricerca sul Cancro (AIRC), work on ARTIST and CLASSIC was supported by the Korean Health Technology R\&D Project, Ministry of Health \& Welfare, Republic of Korea.

AUTHORS' DISCLOSURES OF POTENTIAL CONFLICTS OF INTEREST AND DATA AVAILABILITY STATEMENT

Disclosures provided by the authors and data availability statement (if applicable) are available with this article at DOI https://doi.org/10.1200/ JCO.19.01124

\section{AUTHOR CONTRIBUTIONS}

Conception and design: Filippo Pietrantonio, Rosalba Miceli, Young Woo Kim, Ruth E. Langley, Yoon Young Choi, Sung Hoon Noh, Sung Kim, Maria Di Bartolomeo, David Cunningham, Elizabeth Catherine Smyth Collection and assembly of data: Filippo Pietrantonio, Alessandra Raimondi, Young Woo Kim, Won Ki Kang, Yoon Young Choi, Kyoung-Mee Kim, Federica Morano, Andrew Wotherspoon, Nicola Valeri, MyeongCherl Kook, Ji Yeong An, Giovanni Fucà, Maria Di Bartolomeo, David Cunningham, Jeeyun Lee, Jae-Ho Cheong, Elizabeth Catherine Smyth Data analysis and interpretation: Filippo Pietrantonio, Rosalba Miceli, Alessandra Raimondi, Young Woo Kim, Matthew Guy Nankivell, Andrew Wotherspoon, Nicola Valeri, Heike I. Grabsch, Giovanni Fucà, Sung Hoon 
Noh, Tae Sung Sohn, David Cunningham, Jeeyun Lee, Jae-Ho Cheong, Elizabeth Catherine Smyth

Provision of study material or patients: Filippo Pietrantonio, Young Woo Kim, Won Ki Kang, Kyoung-Mee Kim, Myeong-Cherl Kook, Tae Sung Sohn, Jae-Ho Cheong, Maria Di Bartolomeo

\section{ACKNOWLEDGMENT}

We thank all of the patients who participated in the included studies.

\section{REFERENCES}

1. Siegel RL, Miller KD, Jemal A: Cancer statistics, 2016. CA Cancer J Clin 66:7-30, 2016

2. Bang YJ, Kim YW, Yang HK, et al: Adjuvant capecitabine and oxaliplatin for gastric cancer after D2 gastrectomy (CLASSIC): A phase 3 open-label, randomised controlled trial. Lancet 379:315-321, 2012

3. Cunningham D, Allum WH, Stenning SP, et al: Perioperative chemotherapy versus surgery alone for resectable gastroesophageal cancer. N Engl J Med 355: $11-20,2006$

4. Ychou M, Boige V, Pignon JP, et al: Perioperative chemotherapy compared with surgery alone for resectable gastroesophageal adenocarcinoma: An FNCLCC and FFCD multicenter phase III trial. J Clin Oncol 29:1715-1721, 2011

5. Sakuramoto S, Sasako M, Yamaguchi T, et al: Adjuvant chemotherapy for gastric cancer with S-1, an oral fluoropyrimidine. N Engl J Med 357:1810-1820, 2007

6. Smyth EC, Verheij M, Allum W, et al: Gastric cancer: ESMO clinical practice guidelines for diagnosis, treatment, and follow-up. Ann Oncol 27:v38-v49, 2016

7. National Comprehensive Cancer Network: Gastric cancer guidelines 2015 (version 3.0). http://www.nccn.org/professionals/physician_gls/pdf/gastric.pdf.

8. Cheong JH, Yang HK, Kim H, et al: Predictive test for chemotherapy response in resectable gastric cancer: A multi-cohort, retrospective analysis. Lancet Oncol 19:629-638, 2018

9. Ribic CM, Sargent DJ, Moore MJ, et al: Tumor microsatellite-instability status as a predictor of benefit from fluorouracil-based adjuvant chemotherapy for colon cancer. N Engl J Med 349:247-257, 2003

10. Sargent DJ, Marsoni S, Monges G, et al: Defective mismatch repair as a predictive marker for lack of efficacy of fluorouracil-based adjuvant therapy in colon cancer. J Clin Oncol 28:3219-3226, 2010

11. National Comprehensive Cancer Network: Clinical practice guidelines in oncology: Colon cancer, 2016. http:/Mww.nccn.org/professionals/physician_gls/pdf/colon.pdf

12. Labianca R, Nordlinger B, Beretta GD, et al: Early colon cancer: ESMO clinical practice guidelines for diagnosis, treatment, and follow-up. Ann Oncol 24: vi64-vi72, 2013

13. Smyth EC, Wotherspoon A, Peckitt C, et al: Mismatch repair deficiency, microsatellite instability, and survival: An exploratory analysis of the medical research council adjuvant gastric infusional chemotherapy (MAGIC) trial. JAMA Oncol 3:1197-1203, 2017

14. Choi YY, Kim H, Shin SJ, et al: Microsatellite instability and programmed cell death-ligand 1 expression in stage II/III gastric cancer: Post hoc analysis of the CLASSIC randomized controlled study. Ann Surg 270:309-316, 2018

15. Miceli R, An J, Di Bartolomeo M, et al: Prognostic impact of microsatellite instability in Asian gastric cancer patients enrolled in the ARTIST trial. Oncology 97:38-43, 2019

16. Lee J, Lim DH, Kim S, et al: Phase III trial comparing capecitabine plus cisplatin versus capecitabine plus cisplatin with concurrent capecitabine radiotherapy in completely resected gastric cancer with D2 lymph node dissection: The ARTIST trial. J Clin Oncol 30:268-273, 2012

17. Bajetta E, Floriani I, Di Bartolomeo M, et al: Randomized trial on adjuvant treatment with FOLFIRI followed by docetaxel and cisplatin versus 5-fluorouracil and folinic acid for radically resected gastric cancer. Ann Oncol 25:1373-1378, 2014

18. Morano F: Microsatellite instability (MSI) in resected gastric cancer: Correlation with clinicopathologic and molecular profile. A translational analysis of ITACA-S Study. LBA3256 XX Congresso Nazionale AIOM 2018 - Rome, 2018

19. American Joint Committee on Cancer: TNM Staging Manual (ed 7)

20. Freeman GH, Halton JH: Note on an exact treatment of contingency, goodness of fit and other problems of significance. Biometrika 38:141-149, 1951

21. Durrleman S, Simon R: Flexible regression models with cubic splines. Stat Med 8:551-561, 1989

22. Di Bartolomeo M, Pietrantonio F, Pellegrinelli A, et al: Osteopontin, E-cadherin, and $\beta$-catenin expression as prognostic biomarkers in patients with radically resected gastric cancer. Gastric Cancer 19:412-420, 2016

23. Cancer Genome Atlas Research Network: Comprehensive molecular characterization of gastric adenocarcinoma. Nature 513:202-209, 2014

24. An JY, Kim H, Cheong JH, et al: Microsatellite instability in sporadic gastric cancer: Its prognostic role and guidance for 5-FU based chemotherapy after RO resection. Int J Cancer 131:505-511, 2012

25. Sinicrope $F A$, Shi $Q$, Allegra CJ, et al: Association of DNA mismatch repair and mutations in BRAF and KRAS with survival after recurrence in stage III colon cancers: A secondary analysis of 2 randomized clinical trials. JAMA Oncol 3:472-480, 2017

26. Innocenti F, Ou FS, Qu X, et al: Mutational analysis of patients with colorectal cancer in CALGB/SWOG 80405 identifies new roles of microsatellite instability and tumor mutational burden for patient outcome. J Clin Oncol 37:1217-1227, 2019

27. Shitara K, Özgüroğlu M, Bang YJ, et al: Pembrolizumab versus paclitaxel for previously treated, advanced gastric or gastro-oesophageal junction cancer (KEYNOTE-061): A randomised, open-label, controlled, phase 3 trial. Lancet 392:123-133, 2018

28. Ohtsu A, Shah MA, Van Cutsem E, et al: Bevacizumab in combination with chemotherapy as first-line therapy in advanced gastric cancer: A randomized, double-blind, placebo-controlled phase III study. J Clin Oncol 29:3968-3976, 2011

29. Wilke H, Muro K, Van Cutsem E, et al: Ramucirumab plus paclitaxel versus placebo plus paclitaxel in patients with previously treated advanced gastric or gastrooesophageal junction adenocarcinoma (RAINBOW): A double-blind, randomised phase 3 trial. Lancet Oncol 15:1224-1235, 2014

30. Lin SJ, Gagnon-Bartsch JA, Tan IB, et al: Signatures of tumour immunity distinguish Asian and non-Asian gastric adenocarcinomas. Gut 64:1721-1731, 2015

31. Umar A, Boland CR, Terdiman JP, et al: Revised Bethesda guidelines for hereditary nonpolyposis colorectal cancer (Lynch syndrome) and microsatellite instability. J Natl Cancer Inst 96:261-268, 2004

32. Kim KJ, Lee TH, Cho NY, et al: Differential clinicopathologic features in microsatellite-unstable gastric cancers with and without MLH1 methylation. Hum Pathol 44:1055-1064, 2013

33. Sargent DJ, Shi Q, Yothers G, et al: Prognostic impact of deficient mismatch repair (dMMR) in 7,803 stage II/III colon cancer (CC) patients (pts): A pooled individual pt data analysis of 17 adjuvant trials in the ACCENT database. J Clin Oncol 32, 2014 (suppl; abstr 3507).

34. Fuchs CS, Doi T, Jang RW, et al: Safety and efficacy of pembrolizumab monotherapy in patients with previously treated advanced gastric and gastroesophageal junction cancer: Phase 2 clinical KEYNOTE-059 trial. JAMA Oncol 4:e180013, 2018 
AUTHORS' DISCLOSURES OF POTENTIAL CONFLICTS OF INTEREST

Individual Patient Data Meta-Analysis of the Value of Microsatellite Instability As a Biomarker in Gastric Cancer

The following represents disclosure information provided by authors of this manuscript. All relationships are considered compensated. Relationships are self-held unless noted. I = Immediate Family Member, Inst = My Institution. Relationships may not relate to the subject matter of this manuscript. For more information about ASCO's conflict of interest policy, please refer to www.asco.org/rwc or ascopubs.org/jco/site/ifc.

Filippo Pietrantonio

Consulting or Advisory Role: Amgen, Merck Serono, Bayer, Eli Lilly, Sanofi, Roche, SERVIER

\section{Ruth E. Langley}

Consulting or Advisory Role: Bayer Schering Pharma (Inst)

Research Funding: Bayer Schering Pharma (Inst)

Federica Morano

Honoraria: SERVIER

Travel, Accommodations, Expenses: Sanofi, SERVIER

Andrew Wotherspoon

Consulting or Advisory Role: Celgene

Travel, Accommodations, Expenses: Pfizer

Nicola Valeri

Speakers' Bureau: Bayer, Pfizer, Eli Lilly, Merck

Travel, Accommodations, Expenses: Menarini Silicon Biosystems

Heike I. Grabsch

Honoraria: MSD Oncology
Maria Di Bartolomeo

Honoraria: Eli Lilly, MSD Oncology, SERVIER

Consulting or Advisory Role: Eli Lilly, MSD Oncology

Research Funding: Eli Lilly (Inst)

Travel, Accommodations, Expenses: Roche, Sanofi

David Cunningham

Research Funding: AstraZeneca (Inst), Amgen (Inst), Sanofi (Inst), Merrimack (Inst), Celgene (Inst), Medlmmune (Inst), Bayer (Inst), 4SC (Inst), Clovis Oncology (Inst), Eli Lilly (Inst), Janssen (Inst), Merck (Inst)

\section{Jae-Ho Cheong}

Consulting or Advisory Role: ImmunoMet therapeutics

Research Funding: ImmunoMet Therapeutics

Elizabeth Catherine Smyth

Consulting or Advisory Role: Astellas Pharma, Celgene, SERVIER, Bristol-Myers Squibb

Patents, Royalties, Other Intellectual Property: Patent No: 1716712.3 pending Travel, Accommodations, Expenses: Bristol-Myers Squibb, SERVIER

No other potential conflicts of interest were reported. 